\title{
PAQMAN: Protein-nucleic acid affinity quantification by MAss spectrometry in nuclear extracts
}

\author{
Cathrin Gräwe, Matthew M. Makowski, Michiel Vermeulen* \\ Department of Molecular Biology, Faculty of Science, Radboud Institute for Molecular Life Sciences, Oncode Institute, Radboud University Nijmegen, 6525 GA Nijmegen, \\ The Netherlands
}

\section{A B S T R A C T}

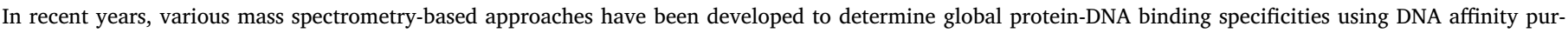

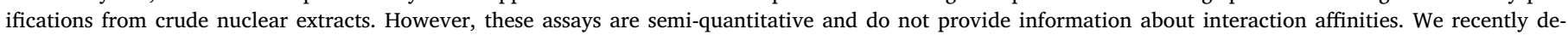

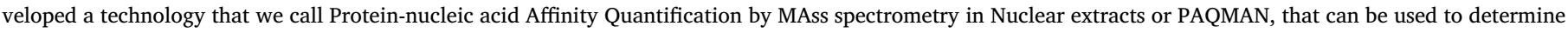

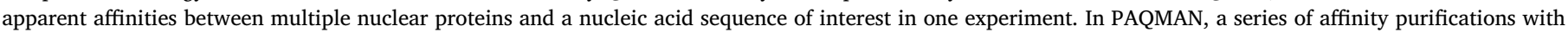

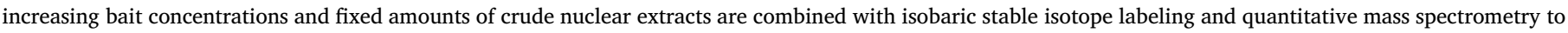

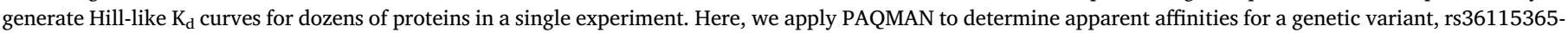

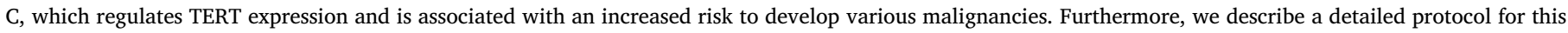
method including important quality checks.

\section{Introduction}

The human genome contains a large number of non-coding elements such as promoters and enhancers, which play an important role in regulating gene expression in a dynamic and spatio-temporal controlled manner, for example during embryonic development but also during adult life. These regulatory elements contain motifs that are recognized and bound by proteins, including transcription factors. Transcription factors can subsequently recruit or inhibit the recruitment of other transcription factors or chromatin modifying enzymes, thereby regulating gene expression $[1,2]$. In recent years, it became apparent that mutations and single nucleotide polymorphisms (SNPs) can influence transcription factor binding in regulatory elements [3]. Therefore, deciphering the molecular mechanisms of transcription factor binding across the genome is not only essential from a fundamental point of view, but is also important to unravel molecular mechanisms of disease.

In recent years, many techniques have been developed to study the complex interplay between proteins and DNA. These techniques are mainly semi-quantitative and provide information about the specificity but not the affinity of an interaction [4]. Specificity refers to the relative strength of a protein-DNA interaction, whereas affinity refers to the absolute strength of this interaction. Combined information regarding specificity and affinity in vitro can help to predict if and when a specific interaction between a transcription factor and DNA occurs in vivo [1].
The binding affinity of a protein to its ligand, in this case DNA or other nucleic acids, can be described by using the dissociation constant $\left(\mathrm{K}_{\mathrm{d}}^{\mathrm{app}}\right)$. The $\mathrm{K}_{\mathrm{d}}^{\text {app }}$ is a numeric representation of the strength with which a protein binds to a given DNA motif, usually expressed in $\mathrm{M}$ (moles/liter). One way to obtain the $\mathrm{K}_{\mathrm{d}}$ is to determine the concentration of DNA that is required to bind half of the total amount of a certain protein, which is equal to the $\mathrm{K}_{\mathrm{d}}$ [5]. Several methods have been developed to determine the binding affinity of proteins to DNA. However, most of these techniques are laborious due to the use of purified recombinant proteins, which can only be investigated on a case-by-case basis [6,7]. However, in recent years, several techniques have been developed that can be used to determine binding affinities on a larger scale $[8,9]$. In addition, mass spectrometry-based methods have been developed that can be used to obtain information concerning absolute affinities between proteins and small molecules $[10,11]$. Recently, we adapted these methods for protein-nucleic acid interactions [12]. We name this method Protein-nucleic acid Affinity Quantification by MAss spectrometry in Nuclear extracts or PAQMAN. PAQMAN can be used to determine dozens of apparent affinities between proteins and a particular DNA sequence of interest in the context of crude nuclear extracts. Here, we applied PAQMAN to study a major oncogenic driver event, which enables replicative immortality, the transcriptional reactivation of telomerase reverse transcriptase or TERT. In previous work, we identified a SNP in an enhancer of the TERT gene, which is associated with altered TERT expression and an increased risk to develop various

\footnotetext{
* Corresponding author.

E-mail address: michiel.vermeulen@science.ru.nl (M. Vermeulen).
} 
malignancies. A PAQMAN experiment for this SNP revealed that ZNF148 interacts with the SNP with a high affinity. This protein was previously shown to regulate TERT expression in a SNP dependent manner, thus illustrating the usefulness of PAQMAN to identify transcription factors that are likely to interact with functional DNA elements in vivo. Furthermore, we provide a detailed protocol to perform a PAQMAN experiment, including important suggested quality checks.

\section{Materials}

\subsection{Buffers for nuclear protein extraction}

\begin{tabular}{ll}
\hline Buffer & Reagents (final concentration) \\
\hline \multirow{2}{*}{ Buffer A } & $10 \mathrm{mM} \mathrm{Hepes} \mathrm{KOH} \mathrm{pH} 7.9$ \\
& $1.5 \mathrm{mM} \mathrm{MgCl}_{2}$ \\
& $10 \mathrm{mM} \mathrm{KCl}$ \\
& Additives: \\
& $0.15 \%$ NP-40* \\
& EDTA-free Complete Protease Inhibitor (Roche-04693132001)* \\
& $420 \mathrm{mM} \mathrm{NaCl}$ \\
& $20 \mathrm{mM}$ Hepes KOH pH 7.9 \\
& $20 \%$ v/v glycerol \\
& $2 \mathrm{mM}$ MgCl \\
Buffer C & $0.2 \mathrm{mM}$ EDTA \\
& $0.1 \%$ NP-40* \\
& EDTA-free Complete Protease Inhibitor (Roche)* \\
& $0.5 \mathrm{mM}$ dithiothreitol (DTT)* \\
&
\end{tabular}

\subsection{Materials for nuclear protein extraction}

\begin{tabular}{ll}
\hline Buffer & $\begin{array}{l}\text { Reagents (final con- } \\
\text { centration) }\end{array}$ \\
\hline $\begin{array}{l}\text { Phosphate Buffered Saline (PBS) } \\
\text { Dulbecco's Phosphate Buffered Saline (DPBS) without Cal- } \\
\text { cium or Magnesium }\end{array}$ & Lonza (17-512F) \\
\hline
\end{tabular}

\subsection{Buffers for PAQMAN}

\begin{tabular}{ll}
\hline Buffer & Reagents (final concentration) \\
\hline \multirow{2}{*}{ TE Buffer (pH 8.0) } & $10 \mathrm{mM}$ Tris \\
& $1 \mathrm{mM}$ EDTA \\
2X Oligo Annealing Buffer & $100 \mathrm{mM} \mathrm{NaCl}$ \\
& $20 \mathrm{mM}$ Tris, pH 8.0 \\
& $2 \mathrm{mM} \mathrm{EDTA}$ \\
& $1 \mathrm{M} \mathrm{NaCl}$ \\
DNA Binding Buffer & $10 \mathrm{mM}$ Tris, pH 8.0 \\
& $1 \mathrm{mM}$ EDTA \\
& $0.05 \%$ NP-40* \\
& $150 \mathrm{mM}$ NaCl \\
& $50 \mathrm{mM}$ Tris, pH 8.0 \\
Protein Incubation Buffer & EDTA-free Complete Protease Inhibitor (Roche)* \\
& $0.25 \%$ NP-40* \\
& $1 \mathrm{mM}$ Tris(2-carboxyethyl)phosphine (TCEP)* \\
& $150 \mathrm{mM}$ NaCl \\
& $100 \mathrm{mM}$ TEAB \\
& $20 \%$ MS-Grade Methanol* \\
Washing Buffer & $80 \mathrm{mM}$ TEAB* \\
& $10 \mathrm{mM}$ TCEP* \\
\hline
\end{tabular}

\subsection{Materials for DNA pulldowns}

\begin{tabular}{ll}
\hline Material & Manufacturer \\
\hline DNA baits & $\begin{array}{l}\text { Custom synthesized by Integrated DNA } \\
\text { Technologies (IDT) }\end{array}$ \\
96-well filter plate, $1.2 \mu \mathrm{M}$ pore & Millipore/Merck (MSBVS1210) \\
$550 \mathrm{mM}$ iodoacetamide &
\end{tabular}

Trypsin $(0.1 \mathrm{ug} / \mathrm{ul})$

96 Well Cell Culture Plate $\quad$ Costar $^{\circledast}$ (3599)

Streptavidin-sepharose high per- $\quad$ GE Healthcare (17-5113-01) formance

TMT10plex ${ }^{\mathrm{TM}}$ Isobaric Label Rea- $\quad$ Thermo Scientific ${ }^{\mathrm{TM}}$ gent Set

\subsection{Buffers for StageTipping}

\begin{tabular}{ll}
\hline Buffer & Reagents (final concentration) \\
\hline Buffer A (mass spectrometry-grade) & $0.1 \%$ formic acid \\
Buffer B (mass spectrometry-grade) & $80 \%$ acetonitrile \\
& $0.1 \%$ formic acid \\
\hline
\end{tabular}

\subsection{Materials for StageTipping}

\begin{tabular}{ll}
\hline Material & Manufacturer \\
\hline Solid phase extraction disk, Empore ${ }^{\mathrm{TM}}, \mathrm{C} 18,47 \mathrm{~mm}$ & $3 \mathrm{M}$ \\
\hline
\end{tabular}

*add freshly before use.

\section{PAQMAN protocol}

In PAQMAN, ten DNA affinity purifications are performed with a titration series of predefined oligonucleotide concentrations and a fixed amount of nuclear extract, followed by TMT labeling of bound proteins and identification as well as relative quantification of proteins by mass spectrometry (Fig. 1).

\subsection{Nuclear protein extraction}

UACC903 were cultured as described previously [13]. For one set of ten affinity purifications, $1 \mathrm{mg}$ of nuclear extract is needed. The ratio of cells/nuclear extract may vary depending on the cell type that is used. For example, about $30 \times 10^{6} \mathrm{HeLa}$ cells are needed to obtain $1 \mathrm{mg}$ of nuclear extract.

All steps are performed on ice and with precooled centrifuges.

- Harvest cells in $50 \mathrm{ml}$ tubes and spin them down for $5 \mathrm{~min}$ at $400 \mathrm{~g}$ and $4{ }^{\circ} \mathrm{C}$.

- Wash cells twice with PBS while combining all cells in one $15 \mathrm{ml}$ falcon tube.

- Determine the pellet volume and add 5 volumes of cold Buffer A without additives. Resuspend the cells and incubate them for $10 \mathrm{~min}$ on ice. Afterwards, centrifuge the cells for $5 \mathrm{~min}$ at $400 \mathrm{~g}$ and $4{ }^{\circ} \mathrm{C}$.

- The cell volume should have increased due to osmotic uptake of water. Add two cell pellet volumes of cold Buffer A plus additives (EDTA-free Complete Protease Inhibitors, 0.15\% NP-40). Resuspend cells and transfer the suspension to a dounce homogenizer (type B pestle).

- Perform 40 strokes with the douncer while pausing every ten strokes to cool the sample on ice for $30 \mathrm{~s}$. Afterwards, centrifuge the suspension for $15 \mathrm{~min}$ at $3200 \mathrm{~g}$ and $4{ }^{\circ} \mathrm{C}$.

- The supernatant is the cytoplasmic extract which can be collected in a separate tube. For nuclear protein extraction, continue with the pellet which contains the crude nuclei.

- Wash the pellet once with $5 \mathrm{ml}$ of DPBS, which is mass spectrometry-grade, without resuspending the pellet. Centrifuge for $5 \mathrm{~min}$ at $3200 \mathrm{~g}$ and $4{ }^{\circ} \mathrm{C}$.

- Determine the pellet volume and add 2 volumes of Buffer C. Resuspend the pellet and transfer the suspension to Eppendorf tubes.

- Rotate the suspension for $60 \mathrm{~min}$ at $4{ }^{\circ} \mathrm{C}$. The suspension will become viscous due to the lysis of the nuclei and the release of chromatin. 
1 Preparing nuclear protein extract
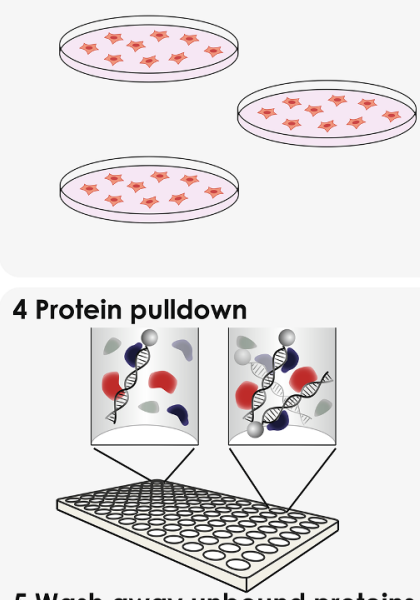

5 Wash away unbound proteins

9 StageTipping
2 Dilution series of oligonucleotides

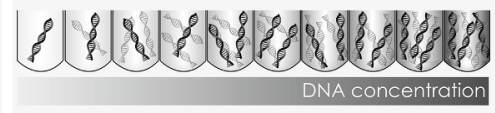

6 Protein digestion

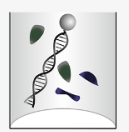

$\downarrow$
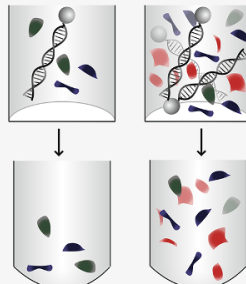

7 Peptide elution

10 Mass spectrometry

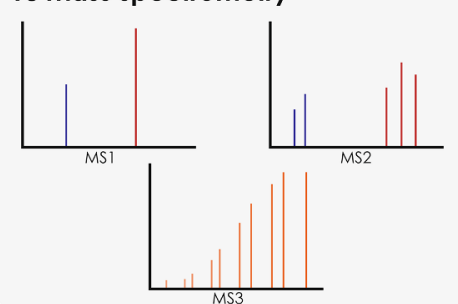

3 Oligo immobilization

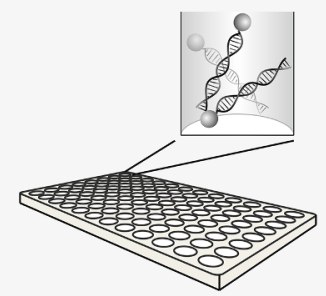

8 TMT labeling

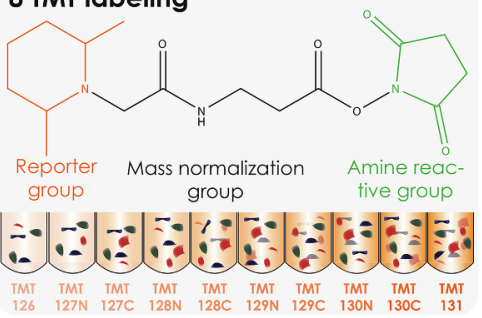

11 Affinity determination

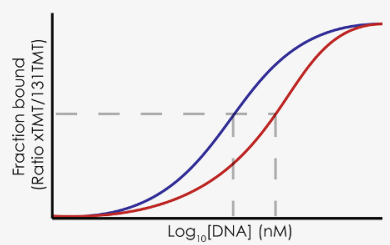

Fig. 1. Schematic overview of the PAQMAN method. 1. Nuclear extract is prepared from cultured cells (Section 3.1). 2 and 3. Custom synthesized 5'-biotinylated DNA oligonucleotides are titrated and immobilized using streptavidin beads on a filter plate (Sections 3.2.1-3.2.4). 4-7. Nuclear extracts are incubated with oligonucleotides, after which unbound proteins are washed away. Bound proteins are on-bead digested with trypsin and eluted from the filter plate (Sections 3.2 .5 and 3.2.6). 8 and 9. Peptides are labeled using isobaric 10-plex TMT after which samples are combined and prepared for mass spectrometry using StageTipping (Sections 3.2.7 and 3.2.8). 10. Peptides are measured by mass spectrometry, TMT reporter ions are quantified in MS3 (Section 3.2.9). 11. $\mathrm{K}_{\mathrm{d}}{ }^{\text {app }} \mathrm{S}$ are determined by calculating the DNA oligonucleotide concentration at which $50 \%$ of the protein is bound to DNA (Section 3.2.10).

- Centrifuge the nuclear suspension and the cytoplasmic extract, if kept, for $30 \mathrm{~min}$ at $20,000 \mathrm{~g}$ and $4{ }^{\circ} \mathrm{C}$.

- Collect the supernatant which contains the nuclear proteins and snap freeze this extract in liquid nitrogen. Store the extract at $-80{ }^{\circ} \mathrm{C}$ until further use.

- If the cytoplasmic extract is kept, add glycerol to a final concentration of $10 \% \mathrm{v} / \mathrm{v}$ and $\mathrm{NaCl}$ to a final concentration of $150 \mathrm{mM}$. Snap freeze in liquid nitrogen and store at $-80{ }^{\circ} \mathrm{C}$ until further use.

- Determine the protein concentration of the extract, for example by using a Bradford assay.

\subsection{PAQMAN assay}

\subsubsection{DNA oligonucleotide design and preparation}

In principle, PAQMAN can be used to determine binding affinities for any (modified) nucleic acid sequence, including RNA or nucleosomal DNA [12]. Here, we use 5' biotin-labeled DNA oligonucleotides containing the SNP rs36115365-C (see Supplementary Table 1). The flanking sequence for a motif of interest can either be random or as here, taken from the genome. Bait length can be adjusted as desired; we have used baits varying in length between 20 and 50 base pairs. In principle, there is no limitation with regard to oligonucleotide bait length, but the longer the sequence the more proteins are pulled down, making it challenging to sequence and quantify all proteins by mass spectrometry.

\subsubsection{Oligonucleotide annealing and dilution}

- For one PAQMAN experiment performed in duplicate, $2.4 \mathrm{nmol}$ of double-stranded oligonucleotides are needed.

- DNA oligonucleotides are custom synthesized (IDT) with the 5' end of the forward strand being biotinylated. Upon arrival, dilute oligonucleotides to $100 \mu \mathrm{M}$ following manufacturer instructions in TE buffer $\mathrm{pH} 8.0$ and store at $-20{ }^{\circ} \mathrm{C}$ until further use.

- Anneal oligonucleotides with a 1.5X molar access of the reverse strand in 2X Annealing Buffer. To generate a $20 \mu \mathrm{M}$ double-stranded DNA oligonucleotide stock, combine $15 \mu \mathrm{l}$ of the $100 \mu \mathrm{M}$ forward oligonucleotide stock with $22.5 \mu \mathrm{l}$ of the $100 \mu \mathrm{M}$ reverse oligonucleotide stock in $37.5 \mu \mathrm{l} 2 \mathrm{X}$ Oligo Annealing Buffer. This is enough to do one titration series.

- Incubate the mixture at $95{ }^{\circ} \mathrm{C}$ for $10 \mathrm{~min}$ to denature oligonucleotides. Then allow the oligonucleotides to anneal by cooling them to room temperature slowly, preferably over night.

- To prepare a $3 \mu \mathrm{M}$ stock of the oligonucleotides, combine $60 \mu \mathrm{l}$ of $20 \mu \mathrm{M}$ annealed oligonucleotides with $340 \mu \mathrm{l}$ DNA Binding Buffer (DBB). This will be the highest concentration in the titration series.

- The dilution series can either be prepared in Eppendorf tubes or in a 96-well plate. Dilute $100 \mu \mathrm{l}$ of the $3 \mu \mathrm{M}$ oligo stock in $200 \mu \mathrm{l}$ of DBB for a three-fold dilution. Prepare a series of nine 3 -fold dilutions by diluting $100 \mu \mathrm{l}$ of the previous oligonucleotide concentration with $200 \mu \mathrm{l}$ DBB, which makes ten samples in total (see scheme below). 


\begin{tabular}{|c|c|c|c|c|c|c|c|c|c|c|}
\hline Well & 1 & 2 & 3 & 4 & 5 & 6 & 7 & 8 & 9 & 10 \\
\hline $\begin{array}{l}\text { Final oligonucleotide concentration } \\
\quad(\mathrm{nM})\end{array}$ & 0.15 & 0.46 & 1.37 & 4.1 & 12.3 & 37 & 111.1 & 333.3 & 1000 & 3000 \\
\hline $\mathrm{DBB}(\mu \mathrm{l})$ & 200 & 200 & 200 & 200 & 200 & 200 & 200 & 200 & 200 & - \\
\hline Volume of oligonucleotide dilution & 100 of well & 100 of well & 100 of well & 100 of well & 100 of well & 100 of well & 100 of well & 100 of well & 100 of well & 300 of $3 \mu \mathrm{M}$ \\
\hline$(\mu \mathrm{l})$ & 2 & 3 & 4 & 5 & 6 & 7 & 8 & 9 & 10 & stock \\
\hline
\end{tabular}

\subsubsection{Preparation of filter plate}

For each experiment, ten affinity purifications are performed consisting of nine data points (dilutions) plus the highest oligo concentration. Every step is performed at $4{ }^{\circ} \mathrm{C}$. Perform each experiment at least in duplicate.

- Place the filter plate on a vacuum-system, for example on the MultiScreen Vacuum Manifold from MERCK. Covering unused wells with PCR tape can improve vacuum.

- Add $50 \mu \mathrm{l}$ of $70 \%$ ethanol to each well and vacuum.

- Wash each well twice with $200 \mu \mathrm{LBB}$ and vacuum. Vacuum after each wash.

\subsubsection{DNA oligonucleotide immobilization on streptavidin coated beads}

- For each affinity purification, use $20 \mu \mathrm{l}$ of Streptavidin-sepharose bead slurry $(10 \mu$ l beads with a total binding capacity of about $3 \mathrm{nmol}$ ) diluted with DBB to a final volume of $200 \mu \mathrm{l}$.

- Add $200 \mu \mathrm{l}$ of the bead mix (10 $\mu \mathrm{l}$ of beads diluted in $200 \mu \mathrm{l} \mathrm{DBB}$ ) to each well. Vacuum.

- Take the filterplate off the vacuum and clean the bottom side with a tissue. Make sure that the bottom side is dry to prevent loss of sample.

- Add $150 \mu \mathrm{l}$ of diluted oligonucleotide mix to the corresponding wells. Use the general scheme described in Section 3.2.2.

- Incubate for at least $1 \mathrm{~h}$ at $4{ }^{\circ} \mathrm{C}$ at $90 \mathrm{rpm}$ on a benchtop mixer, for example on an Orbit P2 Digital Shaker (S2020-P2-B). Alternatively, oligonucleotide immobilization can also be done overnight, which may improve oligonucleotide binding to beads.

- Oligonucleotide depletion after immobilization can be tested by DNA gel electrophoresis (Fig. 2A). To this end, after incubation of the oligonucleotides with the beads in the plate, spin down the plate and collect the flow-through. Then, load a fraction of the flowthrough and the same amount of input oligonucleotide, for example leftover material from the dilution series, on an agarose gel. In this way, depletion of oligonucleotides from the solution on the beads can be manually inspected. Here, we used a $2 \%$ agarose gel and DNA was stained using ethidium bromide. Be aware that a small fraction of excess reverse oligonucleotide will always be visible after oligonucleotide depletion.

\subsubsection{Affinity purification}

- After DNA oligonucleotide immobilization, wash beads once with $200 \mu \mathrm{l}$ DBB and twice with $200 \mu$ l Protein Incubation Buffer (PIB). Vacuum after each wash.

- Take the filter plate off the vacuum and clean the bottom side. Make sure that the bottom side is dry to prevent loss of sample.

- Prepare the nuclear protein solution by adding $100 \mu \mathrm{g}$ of nuclear extract to a final volume of $150 \mu \mathrm{l}$ PIB per pulldown. Make a mastermix for eleven (ten samples plus one extra) affinity purifications to ensure that the exact same amount of proteins is used per pulldown for one experiment. This is important for accurate $\mathrm{K}_{\mathrm{d}}{ }^{\text {app }}$ calculation.

- Apply $150 \mu 1$ nuclear protein solution to beads.

- Incubate for $2 \mathrm{~h}$ at $4{ }^{\circ} \mathrm{C}$ shaking at $90 \mathrm{rpm}$ on a benchtop mixer.

- After incubation, remove unbound proteins by vacuum and wash six times with $200 \mu$ l of Washing Buffer. Quick washes $(<2 s)$ are important to minimize disturbance of the binding equilibrium.

- After the last vacuum, dry the bottom side of the filterplate and place it on a collection plate (96 Well Cell Culture Plate).

\subsubsection{Reduction and alkylation of disulfide bonds followed by trypsin digestion}

Note: The following steps are performed at room temperature.

- Add $50 \mu$ l of Elution Buffer to each well.

- Incubate for $30 \mathrm{~min}$ at room temperature shaking in a humidified incubator. If no humidified incubator is available, use a plastic box with a wet paper tissue.

- Add $5 \mu$ l of $550 \mathrm{mM}$ stock of iodoacetamide (IAA) to a final concentration of $50 \mathrm{mM}$. Incubate for $10 \mathrm{~min}$ at room temperature in the dark and shaking in a humidified incubator.

- Carefully deposit $2.5 \mu \mathrm{l}$ of $0.1 \mu \mathrm{g} / \mu \mathrm{l}$ stock of trypsin on top of the filter in the bottom of each well without damaging the filter $(0.25 \mu \mathrm{g}$ total). Incubate for $1 \mathrm{~h}$ at room temperature shaking in a humidified incubator.

- Elute the sample into a collection plate by centrifuging at $500 \mathrm{~g}$ for 5 min.

- Wash beads by adding $50 \mu$ Elution Buffer to each filter plate well.

- Spin into the same collection plate at $500 \mathrm{~g}$ for $5 \mathrm{~min}$.

- Incubate overnight at room temperature shaking in a humidified incubator.
A

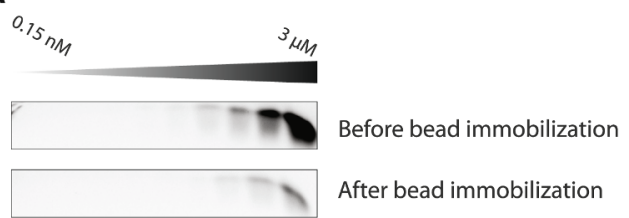

B

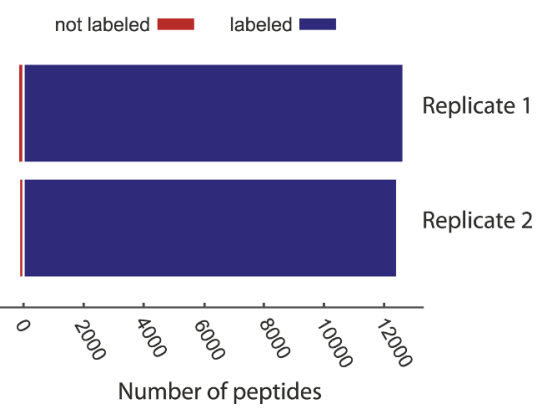

Fig. 2. PAQMAN quality checks. A. Agarose gel electrophoresis of rs36115365-C oligonucleotide titration, before and after binding to streptavidin-sepharose beads, to visualize depletion of the oligonucleotides by bead immobilization. B. TMT labeling efficiency of peptides. For replicate 1, 12,454 out of 12,641 (98.5\%) peptides were labeled, whereas for replicate $2,12,778$ out of 12,426 (97.1\%) peptides were labeled. 


\subsubsection{In-solution TMT labeling}

To quantify the 10 affinity purifications in one LC-MS run, we make use of 10-plex TMT labeling, but other MS2-based isobaric labeling strategies such as iTRAQ [14] or diLeu labeling [15] can also be used. Label-free approaches or MS-1 based labeling strategies such as SILAC are, however, less suitable because of increased mass spectrometry measurement time and increased run-to-run variability.

- Prepare samples for TMT labeling by reducing the sample volume to 10 $\mu \mathrm{l}$ using SpeedVac centrifugation at room temperature.

- Spin down each vial containing $0.8 \mathrm{mg}$ TMT reagent and suspend in $101 \mu \mathrm{l}$ of MS-grade anhydrous acetonitrile.

- Add $10 \mu \mathrm{l}(0.08 \mathrm{mg})$ of TMT reagent to the relevant sample. Apply the TMT label as described in the following scheme:
Samples can be measured on various modern high-resolution mass spectrometers. TMT quantification can be performed at either MS2 or MS3 level, also depending on the instrument that is being used. Although MS2 quantification generally results in higher identification rates, MS3 quantification is more accurate [17]. Therefore, we recommend MS3-based quantification because accurate reporter ion quantification is important for $\mathrm{K}_{\mathrm{d}}$ app calculations. Below we describe settings and parameters for TMT quantification at the MS3 level on an Orbitrap-Fusion mass spectrometer from Thermo Scientific, connected online to an Easy-nLC 1000 system (also Thermo Scientific). $5 \mu$ sample is loaded onto a $30 \mathrm{~cm}$ column with an inner diameter of $75 \mu \mathrm{m}$ (New Objective; heated at $40{ }^{\circ} \mathrm{C}$ ) packed in-house with $3 \mu \mathrm{m}$ Reprosil-Pur C18-AQ beads (Dr Maisch). Tryptic peptides are separated using a gradient from 7 to $15 \%$ Buffer B over 5 min, from 15\% to 35\% Buffer B

\begin{tabular}{|c|c|c|c|c|c|c|c|c|c|c|}
\hline Well & 1 & 2 & 3 & 4 & 5 & 6 & 7 & 8 & 9 & 10 \\
\hline Final oligonucleotide concentration (nM) & 0.15 & 0.46 & 1.37 & 4.1 & 12.3 & 37 & 111.1 & 333.3 & 1000 & 3000 \\
\hline TMT label & 126 & $127 \mathrm{C}$ & $127 \mathrm{~N}$ & $128 \mathrm{C}$ & $128 \mathrm{~N}$ & $129 \mathrm{C}$ & $129 \mathrm{~N}$ & $130 \mathrm{C}$ & $130 \mathrm{~N}$ & 131 \\
\hline
\end{tabular}

Note: The amount of TMT label might need to be adjusted depending on the length of oligonucleotides because more proteins are pulled down when using longer oligonucleotides. Label efficiency can be determined using ProteomeDiscoverer (Thermo Scientific) as described in Section 3.2.10 (Fig. 2B).

- Incubate for $1 \mathrm{~h}$ at room temperature shaking in the dark.

- Add $10 \mu \mathrm{l} 1 \mathrm{M}$ Tris $\mathrm{pH} 8.0$ to quench the reaction and incubate for 30 min shaking at room temperature.

- Combine all 10 related reactions.

\subsubsection{Peptide purification and desalting}

To prepare samples for mass spectrometry analysis, samples are concentrated and desalted using StageTipping [16].

- Add $200 \mu \mathrm{l} 10 \%$ TFA to prepare samples for StageTipping. Check that the $\mathrm{pH}$ is below 2 .

- Prepare the StageTips by picking up two layers of C18 material using a blunt-end syringe and push these into a non-autoclaved 250$\mu l$ pipette tip using a rod. Spin adaptors for the StageTips can be built by removing the lid and bottom of a $0.5-\mathrm{ml}$ Eppendorf tube. Collection tubes can be prepared by cutting of the lid of a 2-ml Eppendorf tube. Place the spin adaptor holding the StageTips into the collection tube.

- Add $50 \mu \mathrm{l} 100 \%$ methanol to the StageTip. Centrifuge for $4 \mathrm{~min}$ at $1.5 \mathrm{~g}$.

- Add $50 \mu \mathrm{l}$ Buffer B to the StageTip. Centrifuge for $4 \mathrm{~min}$ at $1.5 \mathrm{~g}$.

- Add $50 \mu$ l Buffer A to the StageTip. Centrifuge for $4 \mathrm{~min}$ at $1.5 \mathrm{~g}$. Repeat once.

- Load the sample on the StageTip. Centrifuge for $10 \mathrm{~min}$ at $1.5 \mathrm{~g}$. Continue the centrifugation if this is necessary to load the whole sample.

- Add $50 \mu \mathrm{l}$ Buffer A to the StageTip. Centrifuge for $4 \mathrm{~min}$ at $1.5 \mathrm{~g}$.

- StageTips can be stored at $4{ }^{\circ} \mathrm{C}$ until measurement on a mass spectrometer.

\subsubsection{Mass spectrometry}

- Elute the sample from the StageTip using $30 \mu \mathrm{l}$ of Buffer B. Reduce the sample volume to $5 \mu \mathrm{l}$ by SpeedVac centrifugation at room temperature. Afterwards, add $7.5 \mu$ l Buffer A to a total volume of $12.5 \mu$. The sample is now ready to be measured by liquid chromatography-mass spectrometry (LC-MS). over $214 \mathrm{~min}$, from $35 \%$ to $50 \%$ Buffer over $5 \mathrm{~min}$, and eventually from $50 \%$ to $95 \%$ Buffer B in 1 min followed by 5 additional minutes isocratic flow at $95 \%$ Buffer B (flowrate $200 \mathrm{nl} /$ minute). Tryptic peptides eluting from the LC column are directly sprayed into a Thermo Fusion Tribrid mass spectrometer with the built-in Thermo synchronous precursor selection (SPS)-MS3 method. Full MS scans are recorded in the Orbitrap at 120,000 resolution at a scan range of $380-1500 \mathrm{~m} / \mathrm{z}$. Target values for full MS are set to 2.0e5 AGC target and a maximum injection time of $50 \mathrm{~ms}$. Precursors with a charge between 2 and 7, an intensity threshold of 5.0e3, and dynamic exclusion for $60 \mathrm{~s}$ are selected for MS2 analysis. MS2 is performed in top speed data dependent acquisition mode, with precursor priority given based on highest intensity and after isolation in the quadrupole using an isolation window of $0.7 \mathrm{~m} / \mathrm{z}$ units. We use CID activation at a collision energy of $35 \%$ for fragmentation. Target values for MS2 are set to 1.0e4 AGC target with a maximum injection time of $50 \mathrm{~ms}$. MS2 precursors in the mass range of $400-1200 \mathrm{~m} / \mathrm{z}$ are selected for MS3 analysis using the Thermo TMT reagent isobaric tag loss exclusion property and MS2 precursor ions $18 \mathrm{~m} / \mathrm{z}$ units low and $5 \mathrm{~m} / \mathrm{z}$ units high are excluded. The mass spectrometer is operated for MS3 selection in top data-dependent acquisition mode. MS3 ions are selected with synchronous precursor selection activated for ten precursors. The isolation width of MS and MS2 is set to $2 \mathrm{~m} / \mathrm{z}$. The fragmented ions are detected in the Orbitrap with a resolution of 60,000 and a maximum injection time of $120 \mathrm{~ms}$.

\subsubsection{Data analysis and $K_{d}^{\text {app }}$ calculation}

After mass spectrometry, raw MS spectra can be analyzed using MaxQuant or Proteome Discoverer (Thermo Scientific) to identify and quantify proteins. Here, we provide a workflow for analysis using Proteome Discoverer version 2.2.

- For data analysis in Proteome Discoverer version 2.2 select the processing workflow "PWF_Fusion_TMT_Quan_SPS_MS3_SequestHT_ Percolator" and the consensus workflow "CWF_Comprehensive_ EnhancedAnnotation_Reporter_Quan" with standard settings.

- Set the quantification method to 'TMT 10plex' with the TMT 131 label as control channel.

- Perform the "Spectrum Files RC" and "Sequest HT" searches against a uniprot curated human proteome.

- Add the 6-plex TMT reagent mass (229.163 Da) as dynamic modification on histidine, serine, and threonine. Make sure that the 6plex TMT reagent mass is set as static modification on lysines and on the peptide $\mathrm{N}$-terminus.

- Add a file containing contaminants in the section "ProteinMarker". 
A

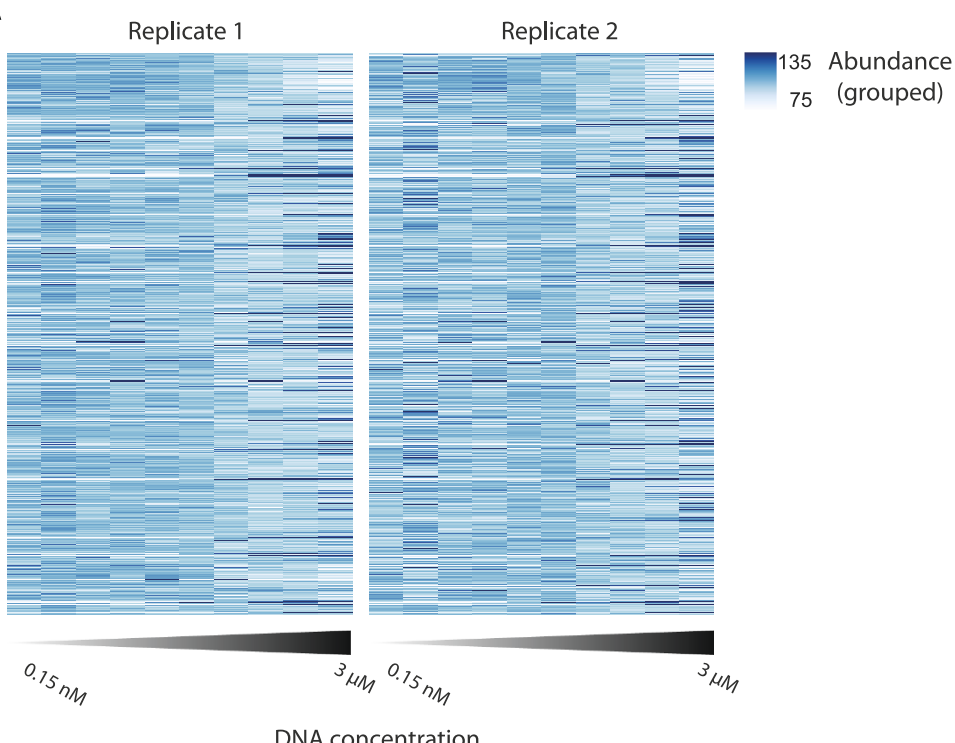

B

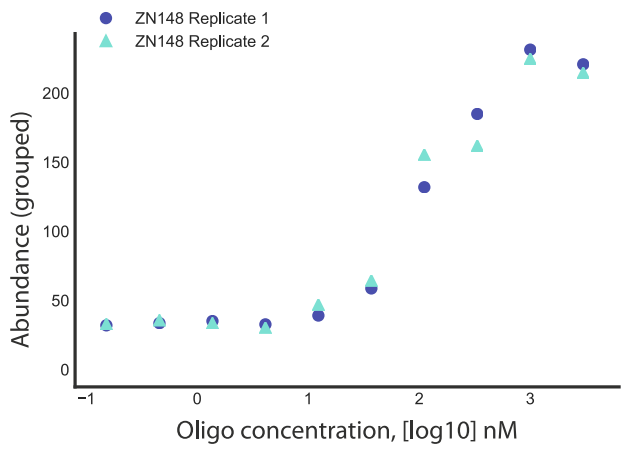

D

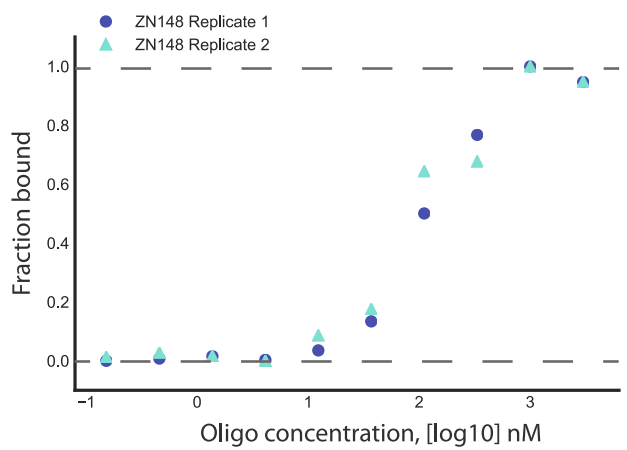

2. Scale Fraction bound between 0 and 1
C

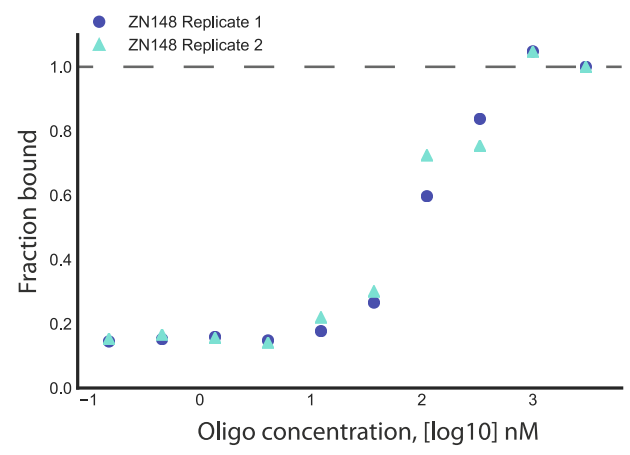

1. Calculate fraction bound

E

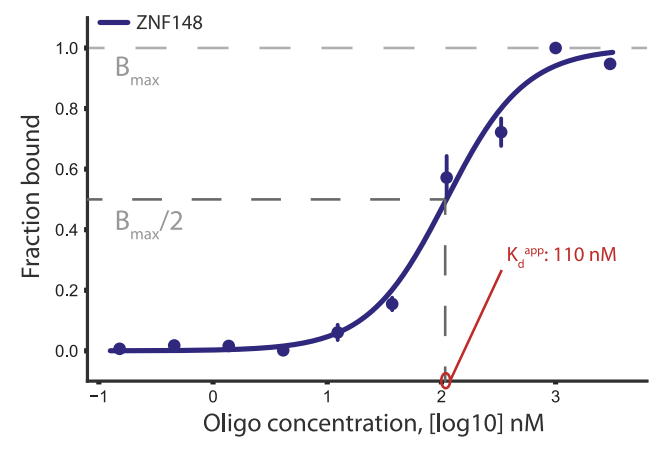

3. Calculate $\mathrm{K}_{\mathrm{d}}^{\text {app }}$

Fig. 3. Data analysis pipeline. A. Grouped abundances obtained from ProteomeDiscoverer 2.2 for proteins identified in both replicates. B. Grouped abundance for ZNF148. C. Fraction bound calculated using grouped abundances. The highest titration point was used as references because binding is saturated at this point. D. Fraction bound is scaled between 0 and 1. E. A Hill-like curve is fitted to determine apparent binding affinities by calculating $\mathrm{K}_{\mathrm{d}}{ }^{\text {app }}$, which is equivalent to the DNA concentration where half of the maximal protein fraction is bound (indicated in red). (For interpretation of the references to colour in this figure legend, the reader is referred to the web version of this article.)

This file can be obtained from the MaxQuant software suite at www. MaxQuant.org.

- When analysis is finished, export the results by selecting 'Proteins' and create a tab limited text file with $\mathrm{R}$ friendly headers.

- TMT labeling efficiency can be tested by analyzing the raw data as described above with the difference that 6-plex TMT reagent mass is added as dynamic modification on lysines and the peptide $\mathrm{N}$-terminus. Labeling efficiency is calculated by comparing the total amount of identified peptides with peptides modified by TMT.

The binding curves and apparent binding affinities can be calculated as described below. To calculate the apparent binding affinities, ratios 
are used. The highest concentration of bait used is $3 \mu \mathrm{M}$, labeled with the TMT reporter ion 131 .

- For further downstream analysis, only 'MasterProteins' are used that are not marked as contaminant (based on the predefined list used in Proteome Discoverer).

- Most proteins are background binders or bind with a low affinity and therefore show no binding curve (Fig. 3A, Supplementary Table 2). However, some proteins bind with high affinity and show a Hill-like curve in the used concentration range (Fig. 3B).

- To obtain the fraction bound for identified proteins, ratios of protein binding are calculated relative to the highest titration point $(3 \mu \mathrm{M}$, TMT 131) (Fig. 3C). Theoretically, the binding at this concentration is saturated for proteins that bind to the oligonucleotide bait with an apparent affinity up to $500 \mathrm{nM}$. Here, we used the ratios based on the normalized abundances that were calculated by Proteome Discoverer.

- Normalize protein ratios by min-max scaling between 0 and 1 (Fig. 3D).

- To generate protein binding curves, fit a Hill-like curve: $\Theta=\frac{1}{\left(\frac{\mathrm{KdApp}}{[L]}\right)^{n}+1}$, where $\Theta$ is the fraction of bound protein, $[L]$ is the bait concentration, $\mathrm{K}_{\mathrm{d}}$ app is the apparent dissociation constant, and $n$ is the Hill coefficient.

- Fit the parameters $\mathrm{K}_{\mathrm{d}}{ }^{\text {app }}$ and $n$ using non-linear least squares with the mean $\Theta$ of each duplicate, with initial parameter estimates of $\mathrm{K}_{\mathrm{d}}{ }^{\text {app }}=100$ and $\mathrm{n}=1$. Plot the binding curves as the mean of replicates plus and minus error bars representing the standard error of the mean. Here, we calculated the standard errors using bootstrapping in the python package seaborn (Fig. 3E).

- The fit of binding curves should be tested to avoid artificial curve- fitting of proteins that do not show binding kinetics. Here, we suggest the following quality checks described below: for analysis, only proteins for which all nine ratios are determined in all replicates are included. After curve fitting, obtained data is filtered by goodness of fit of the Hill-like curve, where an $r^{2}$ value of 0.95 for a linear regression between the fit binding model and the measured data is required. Furthermore, a predicted fraction bound of $<0.25$ for the lowest titration point and more than 0.75 for the highest titration point is required. Care should be taken to not over fit a curve such that the data points appear to reach saturation when this effectively is not the case when looking at the raw data points

\section{Results}

To determine apparent binding affinities for proteins binding to the C-variant of rs36115365, we performed PAQMAN in UACC903 nuclear extract, which is a melanoma cell line. We identified 40 proteins binding with a $\mathrm{K}_{\mathrm{d}}^{\text {app }}<500 \mathrm{nM}$ (Fig. 4A, Supplementary Table 3) of which ZNF148 and VEZF1 were identified previously as specific binders [13]. ZNF148 binds with a higher affinity than VEZF1 (Fig. 4B, C). Other proteins binding with a high affinity were previously identified as non-sequence specific. For ZNF281, previously identified as a specific interactor of the $\mathrm{C}$-variant, $\mathrm{K}_{\mathrm{d}}{ }^{\text {app }}$ could not be calculated, probably because the binding affinity is too low. Another specific binder, ZNF740, was only quantified in one replicate; however, no binding kinetics were observed in this replicate. Follow-up experiments performed previously revealed that ZNF148 regulates TERT expression and telomere length in a SNP-specific manner. This illustrates that PAQMAN experiments are informative and can predict which sequence specific transcription factors identified to interact with a certain motif

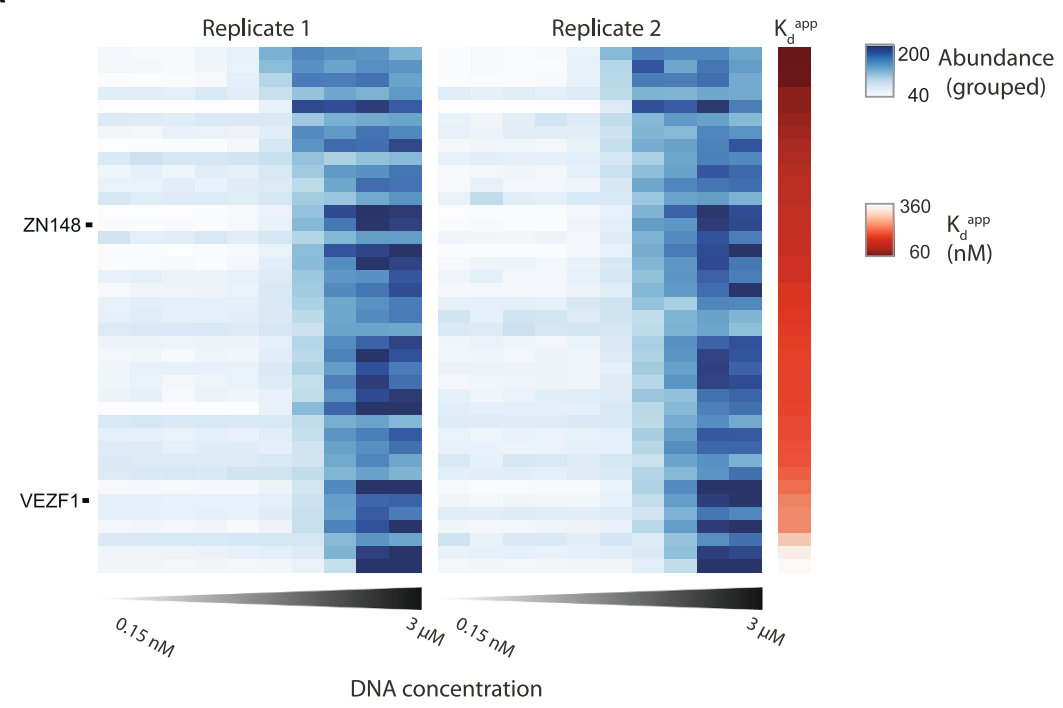

Fig. 4. ZNF148 binds to rs rs36115365-C with high affinity. A. Grouped abundances obtained from ProteomeDiscoverer 2.2 of proteins for which we can calculate a $\mathrm{K}_{\mathrm{d}}{ }^{\text {app }}$, which is plotted in red. B. Binding curve of ZNF148. C. Binding curve for VEZF1. (For interpretation of the references to colour in this figure legend, the reader is referred to the web version of this article.)

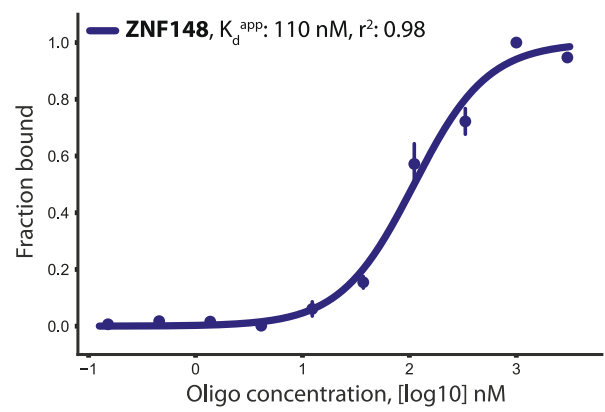

C

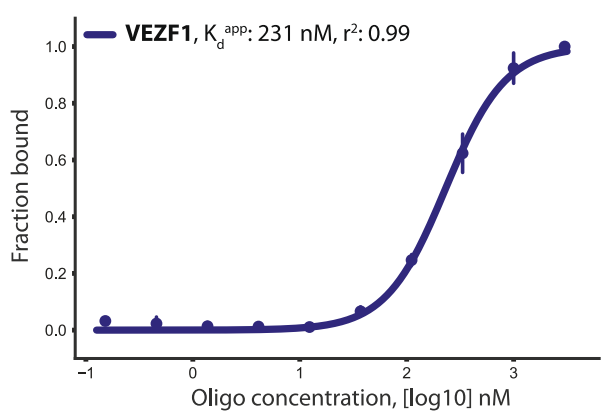


in vitro, regulate gene expression associated with that motif in vivo.

\section{Conclusion and outlook}

Here, we present a detailed protocol for PAQMAN, which is a mass spectrometry-based method that can be used to determine the apparent binding affinities for dozens of proteins to a DNA sequence of interest in a single experiment. Of note, the protocol we present is not limited to protein-DNA interactions but can also be applied to measure affinities between proteins and DNA secondary structures, nucleosomes or RNA [12]. Furthermore, cells treated with a certain stimulus can be used to investigate how this stimulus potentially affects binding affinities.

To illustrate the usefulness of PAQMAN, we profiled a SNP, rs36115365-C, located in an enhancer associated with the TERT gene, which is associated with an increased risk to develop multiple types of cancer. Previous work resulted in the identification of ZNF148 as the transcription factor that, through binding rs36115365-C, drives increased TERT expression. In that study, multiple proteins were identified that bind specifically to the SNP and elaborate follow up experiments were necessary to study these proteins to eventually determine the main regulator, ZNF148 [13]. Here, we determined apparent binding affinities between nuclear proteins and the rs36115365-C SNP and show that amongst all specific rs36115365-C interactors, ZNF148 has the highest apparent binding affinity.

In its current format, PAQMAN is limited to determine apparent binding affinities in the range of up to $500 \mathrm{nM}$ with, in theory, no lower limit. It should be noted that at low bait concentrations, binding competition between multiple nuclear proteins, which display binding affinity for this bait, might occur. Apparent binding constants determined by PAQMAN may thus deviate from measurements done with purified recombinant proteins. However, the apparent binding constants determined by PAQMAN are in principle more informative compared to experiments with single purified recombinant proteins, as these are reflective of the competitive binding environment in vivo. The affinity range of PAQMAN could theoretically be increased to identify lower binding affinities by increasing the concentration of bait DNA. However, in this case the duration of washing steps needs to be decreased to determine low affinities accurately. Low ( $>500 \mathrm{nM}$ ) affinity interactions are typically characterized by a fast off-rate, which means that the product (DNA-protein complex) will dissociate with a half-time of $<1$ s [5]. However, washes after protein extract incubation in PAQMAN normally take about $2 \mathrm{~s}$, thus resulting in disruption of the binding equilibrium for low affinity interactors.

Taken together, information about protein-DNA binding specificity and affinity can now be obtained by mass spectrometry-based proteomic approaches. In the future, we aim to establish a method, which integrates non-isobaric stable isotope labeling and isobaric stable isotope labeling [18] to obtain DNA-protein binding specificity and affinity information in a single LC-MS run.

\section{CRediT authorship contribution statement}

Cathrin Gräwe: Conceptualization, Formal analysis, Investigation,
Data curation, Project administration, Writing - original draft. Matthew M. Makowski: Conceptualization, Supervision. Michiel Vermeulen: Conceptualization, Funding acquisition, Resources, Supervision, Writing - review \& editing.

\section{Acknowledgement}

We would like to thank everyone of the Vermeulen lab for their input and critical feedback. The Vermeulen lab is part of the Oncode Institute, which is partly financed by the Dutch Cancer Society (KWF). Funding for M.M.M. was provided by a grant from the Marie Curie Initial Training Network (ITN) DevCom (FP7, grant number 607142).

\section{Appendix A. Supplementary data}

Supplementary data to this article can be found online at https:// doi.org/10.1016/j.ymeth.2019.12.003.

\section{References}

[1] T. Siggers, R. Gordan, Protein-DNA binding: complexities and multi-protein codes, Nucleic Acids Res. 42 (2014) 2099-2111.

[2] S.A. Lambert, et al., The human transcription factors, Cell 172 (2018) 650-665.

[3] T.N. Cuykendall, M.A. Rubin, E. Khurana, Non-coding genetic variation in cancer, Curr. Opin. Syst. Biol. 1 (2017) 9-15.

[4] N.C. Hubner, L.N. Nguyen, N.C. Hornig, H.G. Stunnenberg, A quantitative proteomics tool to identify DNA-protein interactions in primary cells or blood, J. Proteome Res. 14 (2015) 1315-1329.

[5] T.D. Pollard, A guide to simple and informative binding assays, Mol. Biol. Cell 21 (2010) 4061-4067.

[6] G.D. Stormo, Y. Zhao, Determining the specificity of protein-DNA interactions, Nat. Rev. Genet. 11 (2010) 751-760.

[7] S.J. Maerkl, S.R. Quake, A systems approach to measuring the binding energy landscapes of transcription factors, Science 315 (2007) 233-237.

[8] R. Nutiu, et al., Direct measurement of DNA affinity landscapes on a highthroughput sequencing instrument, Nat. Biotechnol. 29 (2011) 659-664.

[9] C. Jung, et al., True equilibrium measurement of transcription factor-DNA binding affinities using automated polarization microscopy, Nat. Commun. 9 (2018) 1605.

[10] K. Sharma, et al., Proteomics strategy for quantitative protein interaction profiling in cell extracts, Nat. Methods 6 (2009) 741-744.

[11] M. Bantscheff, et al., Chemoproteomics profiling of HDAC inhibitors reveals selective targeting of HDAC complexes, Nat. Biotechnol. 29 (2011) 255-265.

[12] M.M. Makowski, et al., Global profiling of protein-DNA and protein-nucleosome binding affinities using quantitative mass spectrometry, Nat. Commun. 9 (2018) 1653.

[13] J. Fang, et al., Functional characterization of a multi-cancer risk locus on chr5p15.33 reveals regulation of TERT by ZNF148, Nat. Commun. 8 (2017) 15034

[14] S. Wiese, K.A. Reidegeld, H.E. Meyer, B. Warscheid, Protein labeling by iTRAQ: a new tool for quantitative mass spectrometry in proteome research, Proteomics 7 (2007) 340-350.

[15] D.C. Frost, C.J. Rust, R.A.S. Robinson, L. Li, Increased N, N-dimethyl leucine isobaric tag multiplexing by a combined precursor isotopic labeling and isobaric tagging approach, Anal. Chem. 90 (2018) 10664-10669.

[16] J. Rappsilber, Y. Ishihama, M. Mann, Stop and go extraction tips for matrix-assisted laser desorption/ionization, nanoelectrospray, and LC/MS sample pretreatment in proteomics, Anal. Chem. 75 (2003) 663-670.

[17] A. Hogrebe, et al., Benchmarking common quantification strategies for large-scale phosphoproteomics, Nat. Commun. 9 (2018) 1045.

[18] N. Dephoure, S.P. Gygi, Hyperplexing: a method for higher-order multiplexed quantitative proteomics provides a map of the dynamic response to rapamycin in yeast, Sci. Signal. 5 (2012) rs2. 